\title{
DISTRIBUTION OF DEFORMATIONS AND PARAMETERS OF STRESS-STRAIN STATE IN STEEL Kh18N10T
}

\author{
A. M. Rekov, ${ }^{1}$ V. T. Kornienko, ${ }^{1}$ and E. O. Kornienko ${ }^{1}$
}

Translated from Metallovedenie i Termicheskaya Obrabotka Metallov, No. 8, pp. 51 - 55, August, 2013.

\begin{abstract}
Parameters of the laws of distribution of fields of micro- and submicrodeformations in steel Kh18N10T are determined under uniaxial tension of specimens. The components of the plastic strain tensor and their statistics are determined for grain arrays and in the plane of four individual grains. The intensities of the major micro- and submicrodeformations are computed. The correlation relations between the deformations of individual volumes of metal inside grains are estimated. The parameters of the strain-stress states of structural components (grains) of a polycrystal are determined. Qualitative analysis of the microstructural features of deformation of the steel studied is performed.
\end{abstract}

Key words: distribution laws, plastic strain tensor, stress-strain state of microstructure, grains of polycrystals, statistical analysis, structure flaws, slip lines.

\section{INTRODUCTION}

In the processes of pressure treatment of metals various structure flaws, pores and cracks can appear even in early deformation stages. The flaws are caused by local deformation-induced overloads of the microstructure and, to a considerable degree, by inhomogeneity of the properties of individual crystallites and depends on the preceding heat treatment of the material. Single-phase metals, the grains of which differ only in the shape, size and orientation of the crystal lattice with respect to the coordinate system bound to the specimen, have the simplest structure [1]. It is obvious that the mechanical properties of such materials are determined by the evolution of their structure in the course of the deformation. Results of our studies and of other authors show that the variety of the mechanisms of plastic deformation can be represented by two large groups, one of which is related to the intragrain deformation and the other to the deformations over grain boundaries. The topography of submicrodeformations of steel Kh18N10T is presented in [1,2], where their distribution is shown to depend on the boundaries and orientation of individual grains with respect to the axis of the specimen. The deformations of the whole of the specimen (macrodeformations) and micro- and submicrodeformations have been determined in experiments for grain

1 Branch of the Ural Federal University named after the first President of Russia B. N. Yeltsin (FGAOU VPO UrFU), Pervouralsk, Russia (e-mail: arekov@yandex.ru). arrays $(20 \times 20)$ and in the plane of individual grains, respectively. Here and below the deformation levels are classified as in monograph [1].

In addition to quantitative analysis of microstructural features for studying the mechanisms of the development of strain the authors use methods of quantitative statistical metallography. These methods make it possible to take into account the randomness of the properties, stresses and strains typical for polycrystalline materials when estimating their ductility and reliability [1]. Allowance for the inhomogeneity of the properties of a material at the submicrolevel makes it possible to predict the appearance of pores and cracks with sizes commensurable to the size of the grains of the material in the bulk of grains in the deformation process. To determine submicrodeformations we used in the present work the method of measuring of cells of a dividing mesh [3].

The distribution density of micro- and submicrodeformations has been plotted in [4]. We determined there the parameters of the distribution functions of submicrodeformations and obtained estimates of stress-strain states and correlation relations between deformations in the plane of four individual grains at two degrees of deformation of the whole of a specimen, i.e., 6 and $10 \%$.

The distribution functions allow us to determine the relative number of grains and submicrovolumes inside individual grains deformed above some limiting value. Pores nucleate inside these very volumes and microcracks arise. The coefficients of correlation between strains at two neighbor 

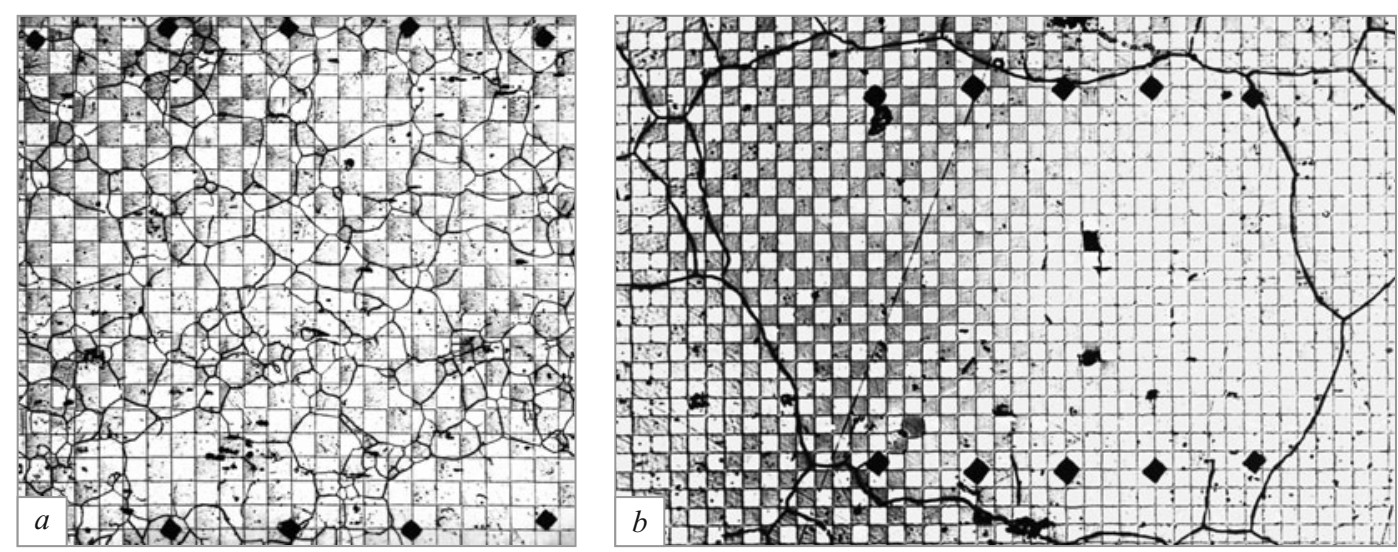

Fig. 1. Regions of the surface of a specimen with dividing mesh of $50(a)$ and $10 \mu \mathrm{m}(b)$ prior to deformation: a) $\times 40 ; b) \times 100$.

points of the surface of the specimen are used to estimate the propagation of high strain over the volume of a grain. The distribution of the random parameter of Nadai-Lode reflects the relative number of grain submicrovolumes that experience the most "rigid" stress-strain state corresponding to uniaxial tension.

The aim of the present work was to study the mechanisms of intragrain deformation in steel Kh18N10T and to estimate statistically the parameters of inhomogeneity of submicrodeformations.

\section{METHODS OF STUDY}

We studied specimens of steel $18 \mathrm{Kh} 10 \mathrm{~T}$ after recrystallization annealing at $1050^{\circ} \mathrm{C}$ with a hold of $12 \mathrm{~h}$ and slow cooling [2]. The boundaries of the austenite grains were determined by etching in standard solutions. The specimens were extended in two stages, i.e., to residual plastic deformations $\varepsilon=5.6$ and $9.8 \%$ (the longitudinal deformations of the studied grains were $\varepsilon^{*}=3.7-12.5 \%$ ). After each stage of extension we estimated the strain-induced microtexture on the surface of the specimen.

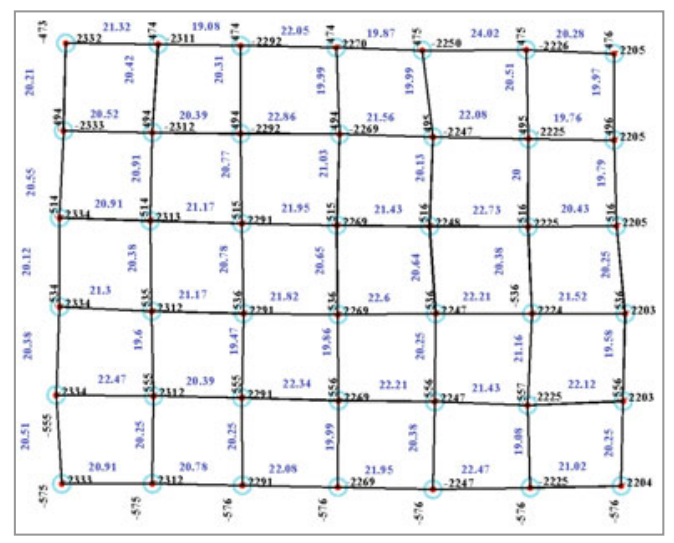

Fig. 2. Fragment of a drawing with measurement results (a replica from monitor screen).
Dividing meshes of a chess type were deposited on the surface of flat specimens by a photographic method [1]. The sizes of a side of a cell of the dividing mesh for determining the microdeformations were assumed to be commensurable to the mean grain size of the material and equal to $50 \mu \mathrm{m}$ (Fig. 1a). The submicrodeformations were determined with the help of a dividing mesh with a cell with a side of $10 \mu \mathrm{m}$ (Fig. 1b).

Four large grains were chosen in the field of the specimen so that the measured cell array was representative enough $(20 \times 20$ cells). We took into account the orientation of the crystallographic planes of the grains with respect to the axis of tension of the specimen $[2,4]$.

The boundaries of the measured arrays were marked with the help of the diamond cutting point of a PMT-3 device.

The coordinates of the nodes of the initial and deformed dividing meshes were measured on the screen of an AUTOCAD system. The photographs of regions of the surface of the specimen magnified under a microscope were transformed into a scanning image with the help of 600-dpi scanner and recorded to a dwg file. The image was scaled. The scale was chosen so that the mean size of the side of the dividing mesh on the screen was $20 \mu \mathrm{m}$. Then the nodes of the cells were marked by reference points (the rings in Fig. 2). The coordinates of the centers of these rings (the red points in Fig. 2) were determined with the help of commands of the AUTOCAD software. The coordinates of the nodes of the mesh and the projections of the lengths of the cell sides on the coordinate axes (the blue figures) were marked in the drawing displayed on the monitor (Fig. 2). The coordinates were used to compute the shear angles and the areas of the deformed cells. The initial value of the length of the side of an undeformed cell of the dividing mesh was determined with allowance for the scale of the image. The results of the measurement were stored as a file text for further processing by a program written in AUTOLISP [3].

The results of the measurements were used to compute the deformations of the cells of the mesh in the plane of the 


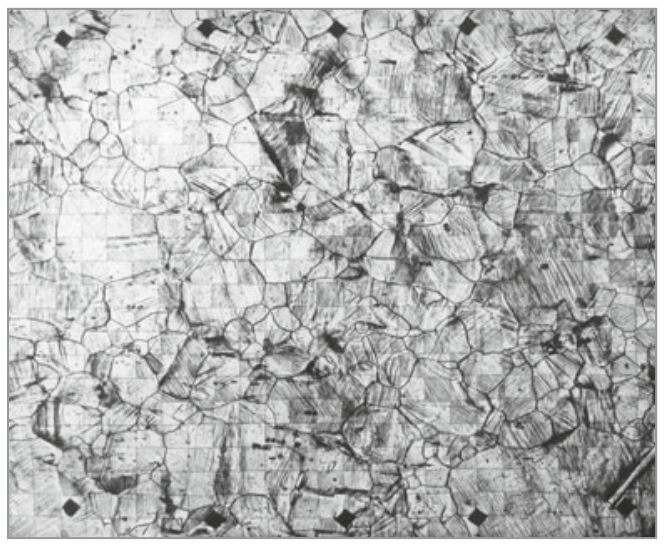

Fig. 3. Microtexture in a region of the surface of a specimen (50- $\mu \mathrm{m}$ dividing mesh, $\varepsilon=9.8 \%), \times 50$.

specimen, i.e., longitudinal deformations $\varepsilon_{11}$ (in the direction of the tension axis) transverse deformations $\varepsilon_{12}$, and shear deformations $\varepsilon_{12}$. These were used to compute the major deformations [5]

$$
\left.\varepsilon_{1,2}=\left[\varepsilon_{11}+\varepsilon_{22} \pm \sqrt{\left(\varepsilon_{11}-\varepsilon_{22}\right)^{2}-\varepsilon_{12}^{2}}\right)\right] / 2 .
$$

Deformation $\varepsilon_{3}$ was determined from the condition of incompressibility of the microvolume of the metal

$$
\varepsilon_{1}+\varepsilon_{2}+\varepsilon_{3}=0
$$

The stress state of individual volumes of the metal was evaluated from the intensity of the major micro- and submicrodeformations

$$
\varepsilon_{\mathrm{i}}^{2}=2\left[\left(\varepsilon_{1}-\varepsilon_{2}\right)^{2}+\left(\varepsilon_{2}-\varepsilon_{3}\right)^{2}+\left(\varepsilon_{3}-\varepsilon_{1}\right)^{2}\right] / 9
$$

and with the help of the Nadai - Lode coefficient $[6,7]$

$$
\chi=\left(2 \varepsilon_{2}-\varepsilon_{1}-\varepsilon_{3}\right) /\left(\varepsilon_{1}-\varepsilon_{3}\right),
$$

where $\varepsilon_{1}>\varepsilon_{2}>\varepsilon_{3}$ are the major micro- and submicrodeformations.

The strain-induced texture of the surface of the specimens was determined using an "Epiquant" microscope.

\section{RESULTS AND DISCUSSION}

The development of the intragrain strain is characterized in the first turn by ordinary nonuniform slip. Several slip systems, twins and other deformation-induced flash can be observed inside one grain (Fig. 3).

Grain No. 1 in Fig. $4 a$ exhibits three systems of ordinary slip. One of them is dominant. All the slip lines are grouped primarily near grain boundaries with different densities. The slip lines are virtually absent in the center of the grain or go at a considerable distance from each other. The slip lines going from the center of the grain to the lower boundary
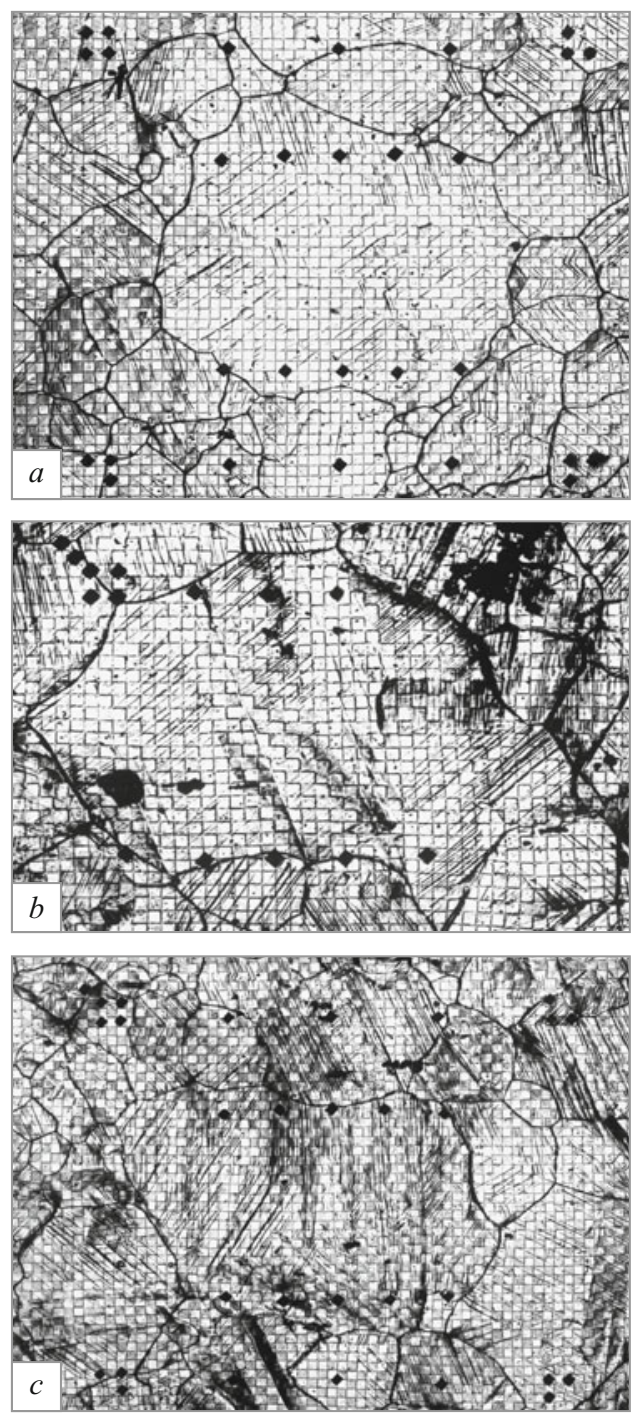

Fig. 4. Grains No. $1\left(\varepsilon^{*}=3.84 \%\right)(a)$, No. $2\left(\varepsilon^{*}=8.3 \%\right)(b)$ and No. $\left.\left.3\left(\varepsilon^{*}=12.5 \%\right)(c): a, c\right) \times 120 ; b\right) \times 130$.

have an implicit form of purely transverse slip. Twin boundaries are discernible but poorly.

Grain No. 2 (Fig. $4 b$ ) is characterized by one system of ordinary slip with flat shear lines intersecting almost the whole of the field. Very small elements of various transverse slip lines and two twins arranged across the direction of the slip lines are observable. On these twins the density of the shear lines differs with respect to their length. Smeared inflection bands go over the larger twin. The inflection bands (narrow) are concentrated over the grain boundaries too. A small third twin is located at the right boundary of the grain and is stretched over the tension axis. The slip lines on it are very dense and a smeared inflection band goes over them. Strain bands are observable at the right boundary of the grain.

On grain No. 3 (Fig. 4c) in the second deformation stage the density of the slip lines gas grown due to the appearance 


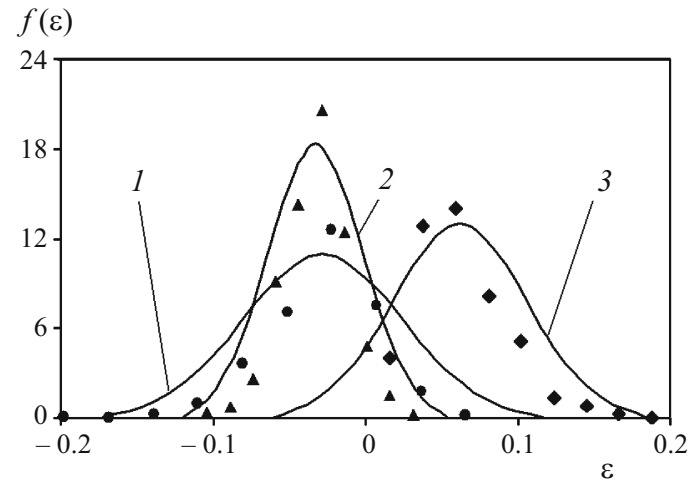

Fig. 5. Distribution densities for major deformations $(\varepsilon=5.6 \%)$ : 1) $\left.\left.\varepsilon_{3} ; 2\right) \varepsilon_{2} ; 3\right) \varepsilon_{1}$.

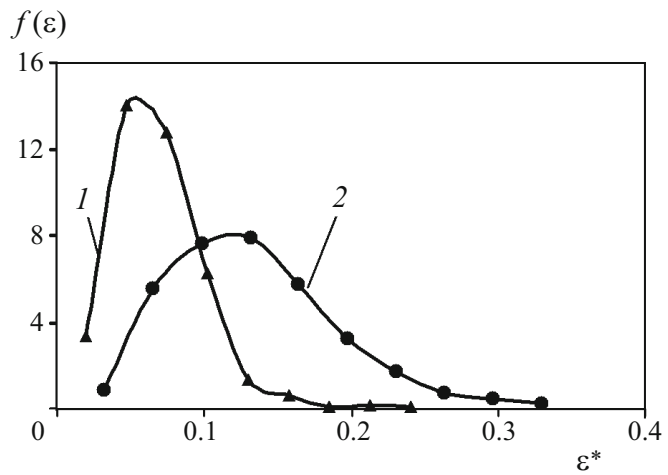

Fig. 6. Distribution densities for the intensity of submicrodeformations due to longitudinal deformation of grains $\varepsilon^{*}=3.74 \%$ (1) and $\varepsilon^{*}=7.32 \%(2)$.

of new and prolongation of old slip traces. The primary systems of ordinary slip are superimposed and form multiple slip. All the slip lines are joined into different groups. Strain bands are located in the groups (especially those strictly perpendicular to the tension axis). The strain bands ore covered with climb bands with separated boundaries. A well discernible small-size inflection band is observed near the lower marks of the field. Some slip systems consist primarily of long slip lines and others have short lines. The density of the slip lines is again different in different regions of the grain. The short slip lines are characterized by a high density of traces.

Similar results have been obtained in the analysis of the strain-induced texture of grain No. 4.

It has been shown in $[1,7,8]$ that in most materials with various lattices, which have been tested at various temperatures the experimentally determined laws of distribution of microdeformations obey a normal distribution law. The distribution densities of the major microdeformations due to uniaxial tension of a specimen of the steel studied are presented in Fig. 5. The specimen is deformed to $\varepsilon=5.6 \%$.

The distribution of the submicrodeformations differs substantially from a normal law (Fig. 6). The parameters of

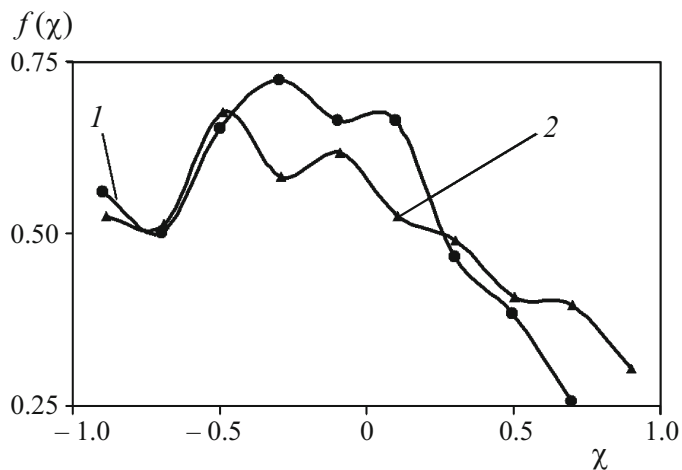

Fig. 7. Distribution densities for Nadai - Lode random coefficients at longitudinal deformations $\varepsilon^{*}=3.74$ (1) and $\varepsilon^{*}=7.32 \%(2)$.

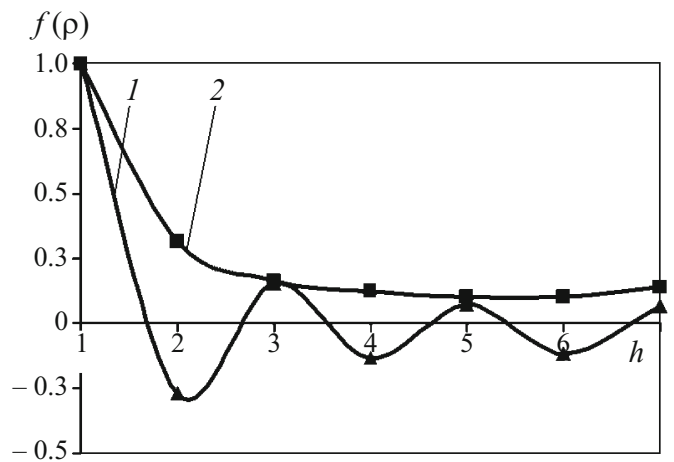

Fig. 8. Correlation functions of submicrodeformations over ( 1 ) and normally to $(2)$ the tension axis ( $h$ is the step of the dividing mesh equal to $10 \mu \mathrm{m})$.

the distribution laws of the submicrodeformations depend on the strain and on the orientation of the crystallographic planes of the grains with respect to the tension axis. The maximum strain the submicrostructure is $\varepsilon^{*}=19 \%$ at macrodeformation $\varepsilon=5.6 \%$ and attains $\varepsilon^{*}=35 \%$ at $\varepsilon=9.8 \%$, i.e., exceeds the mean deformation of the specimen by more than a factor of 3. The most deformed regions of the grain are located primarily over its boundaries oriented at an angle of more than $45^{\circ}$ to the tension axis of the specimen [2].

The stress-strain states of metal volumes inside an individual grain differ substantially from the uniaxial tension at which the tests were performed. Only a relatively low fraction of the volumes $(20-30 \%)$ obeys the stress-strain state matching the Nadai - Lode parameter $\chi=-1$ (uniaxial tension). Individual submicrovolumes of a grain can experience pure shear $(\chi=0)$ and uniaxial compression $(\chi=1)$ (Fig. 7).

Correlation functions give a visual notion of the linear relations between submicrodeformations. The fluctuations of the plot of a correlation function damp down over the tension axis upon growth in the distance $h$ between the cells of the dividing mesh. In the transverse direction the decrease of the linear relation obeys an exponential law. The correlation radius is equal to $5-6$ distances $h$, which corresponds to $50-60 \mu \mathrm{m}$ (Fig. 8). 
The results of the present work are applicable to practical pressure treatment of metals, for example, for designing the routs of production of cold-deformed pipes with allowance for the probabilities of local overloads of the microstructure $[9,10]$.

\section{CONCLUSIONS}

1. The development of nonuniform strain in the plane of individual grains causes inhomogeneity of the fields of plastic submicrodeformations.

2. We have determined the parameters and performed a comparative analysis of one-dimensional and two-point laws of distribution of micro- and submicrodeformations.

3. An experimental distribution of submicrodeformations in the plane of an individual grain can be approximated by a Cauchy function.

4. The fields of submicrodeformations, like the fields of microdeformations, are anisotropic; the sections of normalized autocorrelation functions differ in directions orthogonal with respect to the axis of tension of the specimen.

5. Individual metal volumes in the plane of a grain have different stress-strain states that vary from uniaxial tension to uniaxial compression.

6 . The results of the study can be used for computing the probabilities of the appearance and propagation of flaws in the process of production of pipes and other critical articles.

The study has been performed in accordance with the fundamental state-supported research topic "Experimental and Theoretical Methods of Determination of Nonuniformity of Properties, Stresses and Strains in Microstructure."

\section{REFERENCES}

1. A. A. Vainshtein and A. A. Alekhin, Fundamentals of the Theory of Elasticity and Plasticity with Allowance for Inhomogeneity of the Material [in Russian], UGTU-UPI (2004), 236 p.

2. A. A. Vainshtein, E. O. Kornienko, V. T. Kornienko, et al., Izv. Vysh. Ucheb. Zaved., Chern. Metallurg., No. 6, 63 - 64 (1990).

3. A. M. Rekov, V. T. Kornienko, and É. O. Kornienko, Science and Technology, Vol. 1, Brief Reports of the XXX Russian Workshop Devoted to the 65th Anniversary of the Victory [in Russian], UrO RAN, Ekaterinburg (2010), pp. 179 - 181.

4. A. M. Rekov, V. T. Kornienko, and E. O. Kornienko, "Laws of distribution of deformations and parameters of stress-strain sates of the structural components of steel," Vestn. Nizhegorod. Univ. Im. N. I. Lobachevskogo, No. 4, Part 5, 2457-2459 (2011).

5. A. M. Rekov, A. A. Vainshtein, and V. V. Berezin, "Distribution of major microdeformations" Vetsn. UGTU-UPI: Mekh. Mikroneodnorod. Mater. Razhrush, 22(52), 85 - 88 (2004).

6. A. A. Vainshtein, A. M. Rekov, and V. V. Lapin, "Distribution of microdeformations at boundaries of nonmetallic inclusions," Metalloved. Term. Obrab. Met., No. 10, 27 - 28 (1990).

7. A. A. Vainshtein, A. M. Rekov, and S. A. Ladygin, "Effect of heat treatment modes of titanium on the nonuniformity of microdeformations upon tension," Metalloved. Term. Obrab. Met., No. 8, 17 - 20 (2001).

8. A. A. Vainshtein and A. M. Rekov, "Nonuniformity of the stress-strain state of grains in microstructure at elevated temperatures," Metalloved. Term. Obrab. Met., No. 11, 28 (2001).

9. A. M. Rekov, A. A. Vainshtein, and S. A. Ladygin, "A method for comparing routes of plugless production of pipes with allowance for overloads in microstructure," Chern. Metallurg., Byull. Nauch.-Tekh. Inform., No. 6(1118), 31 - 33 (1992).

10. A. M. Rekov and A. A. Vainshtein, "Estimation of the effect of the drawing mode on the quality of product: the capacity to resist overloads in microstructure," in: Advancements in the Theory and Practice of Pipe Production, Coll. Works [in Russian], GOU VPO “UGTU-UPI," Ekaterinburg (2004), pp. 69 - 71. 